Canadian

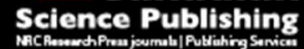

Canadian Journal of Chemistry Revue canadienne de chimie

\title{
Molecular Structure and Melting: Implications for Phase Change Materials
}

\begin{tabular}{|r|l|}
\hline Journal: & Canadian Journal of Chemistry \\
\hline Manuscript ID & cjc-2017-0578.R1 \\
\hline Manuscript Type: & Article \\
\hline Date Submitted by the Author: & 03-Nov-2017 \\
\hline Complete List of Authors: & $\begin{array}{l}\text { Noël, John; Dalhousie University, Chemistry } \\
\text { Kahwaji, Samer; Dalhousie University, Chemistry } \\
\text { White, Mary Anne; Dalhousie University }\end{array}$ \\
\hline $\begin{array}{r}\text { Is the invited manuscript for } \\
\text { consideration in a Special } \\
\text { Issue?: }\end{array}$ & Dalhousie \\
\hline Keyword: & thermodynamics, energy storage, phase change materials \\
\hline
\end{tabular}

\section{SCHOLARONE \\ Manuscripts}


$7{ }^{\mathrm{b}}$ Clean Technologies Research Institute, Dalhousie University, Halifax, Nova Scotia B3H 4R2,

8 Canada

9 *Author for correspondence; email: mawhite@dal.ca

10 


\section{Abstract}

15 Phase change materials (PCM) offer a promising technology for thermal energy storage, load

16 leveling, and peak shifting applications. A desirable PCM has a melting temperature within the

17 temperature boundaries of its application, and a high change in enthalpy on melting. Knowledge

18 of the relationships between these thermodynamic properties and molecular structure would

19 advance informed selection of PCM candidates for a given application. In the present

20 investigation, the relationship between structure (length of alkyl chains) and properties has been

21 investigated for isomeric esters, showing that esters containing longer individual alkyl chains

22 have higher melting temperatures, and higher enthalpy changes on melting. The melting entropy

23 changes, however, are relatively independent of the alkyl chain distribution.

24

25 Keywords:

26 Thermodynamics

27 Energy storage

28 Phase change materials 


\section{Introduction}

One of the barriers to a large-scale shift to renewable energy is the inherent intermittency

32

of renewable energy sources, especially wind and solar, leading to an imbalance between the supply of renewable energy and the demand from the grid. As such, storage technologies are required to make renewable energy available at times when its source is not. Furthermore, energy demand needs to be reduced through energy conservation and increased energy efficiency. While considerable focus is placed on electrical energy, there are gains to be made in terms of thermal energy too, especially when the end use is heat. Solar thermal energy can be used to heat homes and water, but requires energy storage for use when solar flux is low. Approximately $50 \%$ of domestic energy use goes toward heating and cooling of buildings. ${ }^{1}$ As such, reduction of heating and cooling requirements, and increase in insulation efficiency, are attractive targets.

Sensible heat storage materials, using the heat capacity of the material, have been used for millennia. ${ }^{2}$ These are materials with high heat capacity and, once heated, they radiate heat for an extended period. Examples include rocks heated in the sun or with fire, and water. Sensible materials also can be used for cooling, as they absorb relatively large inputs of energy for a given increase in temperature. For example, this is the premise of cold cellars constructed of rock or concrete.

More recently, phase change materials (PCM) have been used for thermal energy storage. ${ }^{2,3,4}$ PCMs store energy via the enthalpy change of a phase transition, typically melting, although solid-solid transitions are also possible. ${ }^{5}$ Over moderate temperature ranges, PCMs can store much more energy per unit mass or volume than a sensible heat storage material. PCMs are used in heat storage applications such as solar thermal hot water ${ }^{6}$ and peak shifting. In the latter case, the PCM is heated/charged using off-peak power, and the heat is recovered later. Similarly, 
53 ice-based PCMs can be solidified using off-peak power and then used later for cooling, in place

54 of conventional air conditioning, throughout the day. ${ }^{2}$ Another important application of PCMs is

55 in moderating temperature swings in a building, ${ }^{7}$ thereby reducing heating and air conditioning

56 requirements. PCMs can be placed within walls, floors, or ceilings ${ }^{8}$ to absorb energy during the

57 warmest parts of the day, before releasing it at night when the temperature has dropped. By

58 doing so, extreme temperature swings can be vastly reduced. ${ }^{9}$

Many materials have been studied for use as PCMs, including salt hydrates, paraffins,

60 fatty acids, and esters. ${ }^{10,11,12}$ A number of materials properties are of concern in the selection of

61 an appropriate PCM for a given application. These requirements include appropriate melting

62 temperature, high enthalpy change of fusion, high heat capacity, high thermal conductivity,

63 controllable hysteresis, low cost, high stability, and low toxicity. ${ }^{2}$ Of these, melting temperature

64 and enthalpy change of fusion are most important. The melting temperature of the PCM must fall

65 within the temperature range of the application: if it does not, the PCM will not change phase,

66 and will act solely as a sensible heat storage material. The enthalpy change of fusion (latent heat)

67 of the material should be as high as possible in order to maximize the energy storage density.

An understanding of the connection between chemical structure and thermodynamic

69 properties would allow for informed selection of potential PCMs. By determining which

70 structural features contribute to high enthalpy change on melting, it would be possible to define

71 which classes of materials hold the most promise for latent heat storage applications.

72 Furthermore, knowledge of the link between molecular features and melting temperatures would

73 allow PCMs within a class of materials to be tailored for specific applications at a variety of

74 temperatures. The main question addressed here is: how does molecular structure influence

75 thermodynamic properties associated with melting? Although the overarching question is 


8

general, in this work we examine a specific example: the impact of carbon atom distribution in linear, saturated esters. For a linear, saturated ester containing 12 carbon atoms, it is possible to form 11 different esters, depending on how the carbon atoms are distributed between the alkyl chains on either side of the bridging oxygen atom. All have the same molecular mass, but their thermodynamic properties differ. Here, the influence of structure on melting temperature, enthalpy change, and entropy change are determined by examining three such esters: ethyl decanoate, butyl octanoate, and hexyl hexanoate. The 12-carbon fatty acid, dodecanoic acid, can be considered as a related case, in which all 12 carbon atoms are part of a single alkyl chain. It is a structural isomer of these esters, and is also included in this analysis.

\section{The Thermodynamics of Melting}

The melting temperature $\left(T_{f u s}\right)$ and the enthalpy change on fusion $\left(\Delta_{f u s} H\right)$ are intimately linked to the entropy change on fusion $\left(\Delta_{f u s} S\right)$ by the equality of the Gibbs energy of the solid and liquid phases, whereby at the melting point equilibrium,

$$
T_{f u s}=\frac{\Delta_{f u s} H}{\Delta_{f u s} S}
$$

Immediately it can be seen that when two materials with equal entropy changes on melting are compared, the more stable solid, i.e. the one with the higher $\Delta_{f u s} H$, will have a higher melting point. ${ }^{13}$ Conversely, for two materials of equal $\Delta_{f u s} H$ and the same absolute entropy of the melt, the lower entropy solid, i.e. the material with higher $\Delta_{f u s} S$, would melt at a lower temperature. Furthermore, for a given melting point, the enthalpy and entropy changes will be directly proportional, and so high $\Delta_{f u s} S$ leads to high $\Delta_{f u s} H$. Therefore, by understanding how structure relates to the thermodynamic properties of a material as it melts, it could be possible to predict 
97 which materials are the best candidates for use as PCMs. Note that the focus here is on molecular 98 materials, as they are one of the common families of PCMs.

The investigation of the relationship between molecular structure and melting has a long history. In 1882, after reviewing information for 15000 compounds, Carnelley, ${ }^{14,15}$ showed that

101

102

103

104

105

106

107

108

109

110

111

112

symmetrical molecules with compact and efficient crystal packing tend to have higher melting points. A similar conclusion was reached more recently by Boese et al. ${ }^{16}$ who showed that the melting point of the symmetrical 1,4-dichlorobenzene $(326 \mathrm{~K})$ is significantly higher than the asymmetrical 1,2- and 1,3-isomers (256 K and $246 \mathrm{~K}$, respectively). It is the efficient packing of symmetrical molecules that leads to a more stable solid, and therefore a high melting point.

Another general finding is that melting temperature increases with molar mass so long as the increase in mass does not significantly change the symmetry or packing. This trend can be observed in the even-numbered alkanes. The melting points of the alkanes increase with the addition of methylene units to the chain, forming two nearly linear trends, one for even number of carbons in the chains and one for odd, reflecting the two types of packing. ${ }^{17,18}$ However, branched alkanes have significantly lower melting points than their corresponding structurally isomeric linear alkanes.

Gilson ${ }^{19}$ compiled entropy changes of fusion for the 358 compounds with listed enthalpy changes of fusion available at that time in the CRC Handbook of Chemistry and Physics, ${ }^{20}$ and found three groupings of materials. The recent compilation by Acree and Chickos ${ }^{21,22}$ of thermodynamic properties for over 7000 organic and organometallic compounds allowed us the opportunity to extend Gilson's study to include >5000 organic compounds, and again we distinguished three groupings (Figure 1). 
The small grouping at the low end, $\Delta_{f u s} S \sim 5$ to $25 \mathrm{~J} \mathrm{~mol}^{-1} \mathrm{~K}^{-1}$, typically includes

120

121

122

123

124 125 126

127 128 129 130 131 135 136 compounds that are disordered crystals of rigid molecules. The disorder already present in the crystalline phase makes the additional entropy gain upon melting relatively small. An example in this category is cubane $\left(\mathrm{C}_{8} \mathrm{H}_{8}\right)$ for which $\Delta_{f u s} S$ is $22 \mathrm{~J} \mathrm{~mol}^{-1} \mathrm{~K}^{-1} \cdot{ }^{23}$ In the crystalline phase of cubane, just below the melting point, the cubane molecules rotate rapidly on their lattice sites, and the entropy of fusion is low because the rotational degrees of freedom are already active in the solid.

Figure 1 also shows a second, and large, grouping of compounds with $\Delta_{f u s} S$ centered at about $58 \mathrm{~J} \mathrm{~mol}^{-1} \mathrm{~K}^{-1}$. These compounds form ordered crystals of rigid molecules. Regardless of composition, materials in ordered crystals all tend to have the same entropy change on transforming from solid to liquid. This generalization was noted more than a century ago, and is known as Walden's rule. ${ }^{24}$ (It is analogous to Trouton's rule, ${ }^{25}$ in which the entropy change on evaporation is almost the same for most materials, due to the very similar increase in disorder on going from the liquid to the vapour.)

Finally, 1780 of these 7038 compounds were identified with high entropy changes of fusion $\left(\Delta_{f u s} S>85 \mathrm{~J} \mathrm{~mol}^{-1} \mathrm{~K}^{-1}\right)$. These are typically non-rigid molecules, including those with long alkyl chains. Upon melting, these materials gain considerable entropy as their non-rigid structures allow them to sample many configurations. From equation 1, it can be seen that these materials with high $\Delta_{f u s} S$ could also have high $\Delta_{f u s} H$, which would make them attractive candidates for PCMs. Many of these compounds, including alkanes and fatty acids, are already being used in heat storage applications.

Special mention should be made of the values of $\Delta_{f u s} H$ and $\Delta_{f u s} S$ for solids in which hydrogen-bonding plays an important role. In this case, typically the melting point is higher than 
142 usual due to the stabilizing influence of the hydrogen-bonding on the crystalline structure, so 143 even if $\Delta_{f u s} S$ falls in the "normal" range, then $\Delta_{f u s} H$ can be exceptionally high (see equation 1 ).

144 Sugar alcohols are one such example, with exceptional promise as phase change materials, if 145 their inherent hysteresis is not an impediment. ${ }^{26}$

Saturated fatty acids are a promising family of PCMs. ${ }^{11,12,27}$ They have high melting

147 enthalpy changes, which increase nearly linearly (with a significant odd/even effect ${ }^{18}$ ) with the 148 length of the carbon chain..$^{21,22}$ Their melting temperatures also increase with increasing chain 149 length, although they plateau above a chain of about 20 methylene units. ${ }^{21,22}$ Fatty acids can be 150 used in energy storage applications at a variety of temperatures by selecting a molecule of the 151 appropriate length. Saturated fatty acids also cycle well ${ }^{11,12}$ and can be produced sustainably ${ }^{28}$. 152 They can be extracted from a variety of renewable sources including plants, such as oil palm, 153 algae, and animal fats. Saturated fatty acids typically have very low embodied energy, and that, 154 coupled with their high energy storage density, means that their energy payback time can be very 155 short when used in devices to store renewable energy. ${ }^{28}$

While pure materials often have excellent thermodynamic properties for use in heat

157 storage applications, mixtures also can be extremely useful, especially to allow applications in 158 temperature ranges where there is no suitable pure material. In a simple ideal eutectic system, 159 addition of one material to another leads to depression of the melting point. The minimum 160 melting temperature is found at the eutectic composition. In thermal storage applications, 161 eutectics can be used to access melting temperatures lower than for pure materials. Using 162 saturated fatty acids as an example, if one wished to store energy at $22{ }^{\circ} \mathrm{C}$ for a temperature 163 moderating application say in residential buildings, the closest melting temperatures one could 164 achieve using pure materials are $16.5^{\circ} \mathrm{C}$ with octanoic acid, or $28.4^{\circ} \mathrm{C}$ with undecanoic acid, 
165 166

167

168

169

170

171

172

173

174

175

176

177

178

179

180

neither very useful. However, the eutectic mixture of undecanoic acid ( $\sim 81.5 \mathrm{~mol} \%)$ and tetradecanoic acid has a very useful melting temperature of $21.7^{\circ} \mathrm{C} .{ }^{29}$ Without the use of eutectics, compromises such as more expensive materials, or materials with lower energy storage density might be required. Furthermore, some fatty acid eutectic mixtures are found in nature. ${ }^{30}$ Such a mixture can be directly extracted and used in its natural proportions, avoiding energyintensive separation processes which would then be followed by re-mixing.

The entropy change for melting eutectic mixtures is greater than for a mixture of the pure compounds, raised by the mixing entropy. For the eutectic mixture of decanoic acid ( $82 \mathrm{~mol} \%)$ and tetradecanoic acid, the measured $\Delta_{f u s} S$ is $96 \mathrm{~J} \mathrm{~mol}^{-1} \mathrm{~K}^{-1}, 31$ i.e., at the high end in Figure 1. However, if $\Delta_{f u s} S$ were to be calculated based on the linear proportionality of the amounts of the pure components and their entropy changes on fusion, ${ }^{31}$ excluding mixing, the expected entropy change would be only $87 \mathrm{~J} \mathrm{~mol}^{-1} \mathrm{~K}^{-1}$. The $\Delta_{f u s} H$ of the same eutectic, however, is $28.2 \mathrm{~kJ} \mathrm{~mol}^{-1}$, ${ }^{31}$ which is very close to the value $\left(28.4 \mathrm{~kJ} \mathrm{~mol}^{-1}\right)$ calculated from the molar percentages of each compound (i.e., rule of mixtures). In this case, $\Delta_{f u s} S$ is higher for the mixture but $\Delta_{f u s} H$ is in proportion to the composition, and therefore (Equation 1), $T_{f u s}$ is depressed, as observed. The rule of mixtures does not always predict $\Delta_{f u s} H$ accurately for eutectics, especially if there are strong interactions between the components of the eutectic in the liquid phase. For example, the succinonitrile-phenanthrene eutectic system shows ${ }^{32} \Delta_{\text {fus }} H$ much larger than would be expected based on the rule of mixtures, likely due to non-ideal behaviour as exemplified by liquid-liquid phase separation.

Inagaki and Ishida ${ }^{33}$ have examined the four six-carbon sugar alcohols: galactitol, mannitol, sorbitol and iditol, both computationally and experimentally, for potential use as PCMs. All four alcohols have the same chemical formulae, and identical, linear carbon 
188 189

backbones. They differ only in stereochemistry, with the hydroxyl groups on the four chiral centers arranged differently. Galactitol and mannitol were found to have high values of $\Delta_{\text {fus }} H$ (60 $\left.\mathrm{kJ} \mathrm{mol}^{-1}, 52 \mathrm{~kJ} \mathrm{~mol}^{-1}\right)$ and $T_{\text {fus }}(460 \mathrm{~K}, 439 \mathrm{~K})$, while sorbitol and iditol were found to have lower values $\left(34 \mathrm{~kJ} \mathrm{~mol}^{-1}, 372 \mathrm{~K}, 31 \mathrm{~kJ} \mathrm{~mol}^{-1}, 353 \mathrm{~K}\right) .^{33}$ The authors ascribe the differences to the distribution of the hydroxyl groups. Sorbitol and iditol have adjacent hydroxyl groups which have large electrostatic repulsions that destabilize the crystalline phase. The decreased stability (increase in enthalpy) of the solid phase produces a smaller change in enthalpy upon melting. Based on additional data for sugar alcohols with carbon backbones of 4 to 8 carbons, three suggestions have been made for selection of sugar alcohols for thermal energy storage applications: $:^{33}$ longer backbones provide higher $\Delta_{\text {fus }} H$; the hydroxyl groups should be well separated in their distribution; and backbones with even numbers of carbons provide higher values of $T_{\text {fus }}$ and thereby higher $\Delta_{\text {fus }} H$.

Stamatiou et al..$^{34}$ examined linear fatty esters for use as PCMs. They showed that both $T_{f u s}$ and $\Delta_{f u s} H$ had strong positive correlations with the molar mass of the ester. In the present work, three linear, 12-carbon esters, and also dodecanoic acid have been examined. These compounds are isomers with linear alkyl chains totalling 12 carbons, differing in their distribution of their 12 carbon atoms between the alkyl chains. The influence of this distribution on $T_{f u s}, \Delta_{f u s} H$, and $\Delta_{f u s} S$ was determined, and provides deeper insight concerning how molecular structure can be used to predict PCM properties, and aid in PCM selection.

\section{Materials and Methods}

Experimental values of $T_{\text {fus }}$ and $\Delta_{\text {fus }} H$ were determined using a TA Instruments Q200 differential scanning calorimeter (DSC). The DSC was calibrated for $T_{\text {fus }}$ and $\Delta_{\text {fus }} H$ at 2 and $10 \mathrm{~K}$ $\min ^{-1}$ scan rates with both mercury and indium standards ${ }^{35}$ before the measurements were taken. 
211 Samples of dodecanoic acid (Aldrich, 98\%), ethyl decanoate (Sigma-Aldrich, > 98\%), butyl

212 octanoate (Aldrich), and hexyl hexanoate (Sigma-Aldrich, $>97 \%$ ) were each analysed three times

213 successively with 2 and then $10 \mathrm{~K} \mathrm{~min}^{-1}$ scan rates. Measurements were made in crimped

214 aluminum pans, under a flow of helium at a rate of $25 \mathrm{~mL} \mathrm{~min}^{-1}$. The sample masses were

215 between 8.5 and $10 \mathrm{mg}$. The values of $T_{f u s}$ were determined from onset temperatures from the 2

$216 \mathrm{~K} \mathrm{~min}^{-1}$ scans. A slow heating rate was used for melting point determination to minimize

217 'thermal lag' in the sample. ${ }^{36}$ Melting temperatures determined by this method typically have

218 uncertainties within $1 \mathrm{~K}$. The values of $\Delta_{f u s} H$ were determined from the $10 \mathrm{~K} \mathrm{~min}^{-1}$ scans. A

219 faster heating rate was used for $\Delta_{f u s} H$ measurement because it results in sharper peaks, reducing

220 the contribution of $\Delta_{f u s} H$ swept into the baseline. A well-calibrated DSC with excellent thermal

221 contact between the sample and pan (as is the case for samples melted into the pan) can give

222 transition enthalpy change values with as little as $~ 5 \%$ uncertainty.

\section{Results and Discussion}

The four compounds analysed all have the molecular formula $\mathrm{C}_{12} \mathrm{H}_{24} \mathrm{O}_{2}$, and the chemical 225 structure $R_{1}-\mathrm{O}-\mathrm{C}(\mathrm{O})-R_{2}$. They differ only in their distribution of methylene units between $R_{1}$ and $226 R_{2}$. At one extreme is dodecanoic acid, in which $R_{1}$ is a proton and all carbon atoms belong to $R_{2}$.

227 The other extreme is hexyl hexadecanoate, in which the carbon backbones are distributed evenly 228 on either side of the bridging oxygen.

\subsection{Melting Temperature}

DSC was used to determine $T_{f u s}$, using a $2 \mathrm{~K} \mathrm{~min}^{-1}$ scan rate (Figure 2). The results are

231 summarized in Table 1. (No solid-solid phase transitions were found in the temperature ranges

232 examined.) Over three melting cycles, there was no observable change in the melting 
233 temperature. In all cases the standard deviation in $T_{f u s}$ was less than $0.1 \mathrm{~K}$. The melting points of

234 these compounds increased with increasing length of $R_{2}$, from $217 \mathrm{~K}$ (hexyl hexanoate) to $316 \mathrm{~K}$

235 (dodecanoic acid). Despite the fact that these molecules are isomers and that all their alkyl

236 chains are linear, it is the longest uninterrupted alkyl chains that produced the highest melting

237 points, likely due to their stability due to maximized van der Waals interactions between the

238 chains. Stamatiou and coworkers ${ }^{34}$ examined the melting points of eight linear fatty ester PCMs,

239 including two structural isomers: ethyl tetradecanoate and butyl dodecanoate. Again, in this case,

240 the ester with the longer uninterrupted alkyl chain, ethyl tetradecanoate, was found to have the

241 highest melting temperature. ${ }^{34}$ From the melting points for 53 linear, saturated esters as compiled

242 by Acree and Chickos, ${ }^{21,22}$ it can be seen that this is, in fact, a general phenomenon. As shown in

243 Figure 3, the plot of $T_{f u s}$ vs. number of carbons has a trend of increasing melting temperature

244 with increasing number of carbons. This is expected given that the molar masses of the esters

245 increase with increasing carbon number, and the number of favourable chain-chain interactions

246 in the solid phase increases as well, thereby increasing the stability of the solid phase and

247 increasing the melting point. At each number of carbon atoms ( $x$-axis in Figure 3 ), however,

248 there is a spread of values for $T_{f u s}$. These different values correspond to different structural

249 isomers of linear esters. As also found here, the highest melting temperatures correspond to the

250 esters with the longest uninterrupted alkyl chains. For example, among the esters for which data

251 are available for 32 carbons, ethyl triacontanoate $(341.5 \mathrm{~K})$ has a significantly higher $T_{f u s}$ than

252 either of tetradecyl octadecanoate $(322.8 \mathrm{~K})$ or octadecyl tetradecanoate (324.4 K). Similarly, at

25326 carbons, methyl pentacosanoate $(332.2 \mathrm{~K})$ has a higher $T_{\text {fus }}$ than hexadecyl decanoate (302.6

$254 \mathrm{~K}) .^{22}$ 
From the present results, while the melting points of hexyl hexanoate and butyl octanoate 256 are separated by only $13 \mathrm{~K}$, and those of butyl octanoate and ethyl decanoate by $22 \mathrm{~K}$, there is a 257 much larger jump of $64 \mathrm{~K}$ between ethyl decanoate and dodecanoic acid. This difference could 258 be due, in part, to the fact that dodecanoic acid forms dimers, hydrogen-bonded through the 259 carboxyl head groups of the molecules. ${ }^{37}$ The dimers are found in the crystalline phase, and 260 persist into the liquid phase. ${ }^{37}$ As such, when dodecanoic acid melts, the dimer is similar to a 24261 carbon molecule (i.e., to tetracosanoic acid), rather than a single 12-carbon molecule, and 262 therefore melts at a rather high temperature. However, the 24 carbon atoms of the dodecanoic 263 acid dimer are distributed between two alkyl chains, and thus dodecanoic acid still has a lower 264 melting point and enthalpy of fusion (Table 1) than tetracosanoic acid (356.5 K, 229.22 J g $\left.{ }^{-1}\right){ }^{22}$

266 The amount of supercooling ranged from 9 to $10 \mathrm{~K}, 12$ to $13 \mathrm{~K}$, and 8 to $10 \mathrm{~K}$ for ethyl 267 decanoate, butyl octanoate, and hexyl hexanoate, respectively. This, in itself, is an important 268 consideration if fatty esters are to be used as PCMs, as the application must have a minimum 269 temperature sufficiently low to nucleate the crystalline phase. Without crystallization, the latent 270 heat which was input in melting cannot be recovered. However, to their benefit, crystallization in 271 these esters occurs at relatively consistent temperatures over several cycles (standard deviation $2721 \mathrm{~K})$. This predictable behaviour would be beneficial should they be employed in a PCM 273 capacity. 
278 alkyl chain length. This finding would seem to indicate that more energy is required to separate

279 single long chains than to separate multiple shorter chains with the same additive length, and that 280 the rankings of $\Delta_{f u s} H$ and $T_{f u s}$ among linear isomers of esters are the same.

The picture becomes slightly less clear when considering the data compiled by Acree and

282 Chickos $^{21,22}$ for 53 linear esters, as presented in Figure 4. The molar melting enthalpy change 283 increases more or less linearly with the overall number of carbon atoms in the ester. This is 284 logical given that each addition of a methylene unit increases the number of chain-to-chain 285 intermolecular interactions which must be separated on melting, each requiring an input of energy. Again, the spread in values at a given number of carbon atoms is due to the different

287 enthalpy changes on fusion for the different isomers. While in many cases the highest enthalpy 288 change on fusion for esters of a given length belongs to the ester with the longest uninterrupted 289 alkyl chain (methyl pentacosanoate, methyl heneicosanoate, methyl tetradecanoate, etc.), this is 290 not always the case. And, in several cases, within esters of a given length, the ordering of the 291 enthalpy values does not match the ordering of the longest individual alkyl chains or melting 292 temperature. Ethyl triancontanoate is the 32-carbon ester with the longest individual alkyl chain 293 for which data are available. It had the highest melting temperature of the 32-carbon esters, but 294 the lowest $\Delta_{f u s} H$ value. Instead, for 32 carbons, it is the ester with the shortest maximum 295 individual alkyl chain and the lowest melting point - tetradecyl octadecanoate - that has the 296 highest $\Delta_{\text {fus }} H$. Among the 26-carbon esters, the highest melting isomer and isomer with the 297 longest single alkyl chain, methyl pentacosanoate, also has the highest $\Delta_{\text {fus }} H$. However, 298 hexadecyl decanoate, the lowest melting isomer, does not have the lowest $\Delta_{f u s} H$. Instead, the 299 lowest $\Delta_{\text {fus }} H$ belongs to ethyl tetracosanoate. Ethyl tetracosanoate had the second highest melting 
300 point, and the second longest individual alkyl chain, and so would have been expected to have 301 had the second highest $\Delta_{f u s} H$.

Note that the data from Acree and Chickos ${ }^{21,22}$ are thoroughly tabulated but not assessed 303 for accuracy. As we have indicated elsewhere, ${ }^{2}$ for some PCMs, widely varying values of 304 physical properties have been reported, and some of the reports are not accurate. For example, 305 Acree and Chickos ${ }^{22}$ present 16 values of $\Delta_{f u s} H$ for dodecanoic acid, ranging from $25.4 \mathrm{~kJ} \mathrm{~mol}^{-1}$ 306 to $44.9 \mathrm{~kJ} \mathrm{~mol}^{-1}$. (We take some comfort that the present value, $36 \pm 1 \mathrm{~kJ} \mathrm{~mol}^{-1}$, agrees well with our own previously published values of $37 \pm 2 \mathrm{~kJ} \mathrm{~mol}^{-1}$ and $35 \pm 3 \mathrm{~kJ} \mathrm{~mol}^{-1} .^{11,12}$ ) In principle, different polymorphs can have different melting properties so that could account for some of the spread of reported properties, but for dodecanoic acid, which has three polymorphs, the other

310 two, A and B, convert to the C-form and only the C-form melts. ${ }^{38}$ Especially when only a single

311 data source is given, only general trends should be concluded from the Acree and Chickos ${ }^{21,22}$

312 data, and specific details would require further experimental investigation.

\subsection{Melting Entropy Change}

The entropies of fusion of the examined isomers (Table 1) are all high in the context of

315 values presented in Figure 1. Hexyl hexanoate has the lowest value of $\Delta_{f u s} S\left(\right.$ and $T_{f u s}$ and $\Delta_{f u s} H$ ).

316 The entropy changes for the other three compounds show a slightly different order from the $T_{f u s}$

317 and $\Delta_{f u s} H$ order, with the highest $\Delta_{f u s} S$ belonging to butyl octanoate, and dodecanoic acid and 318 ethyl decanoate having equal values of $\Delta_{f u s} S$. When also considering the 53 esters from Acree 319 and Chickos (Figure 5), ${ }^{21,22}$ the general trends in $\Delta_{f u s} S$ mirror that of $\Delta_{f u s} H$ : there is a strong linear 320 correlation between $\Delta_{f u s} S$ and the number of carbons, and in most cases, among esters of equal 321 carbon number, the one with the longest unbroken alkyl chain has the highest $\Delta_{f u s} S$. The 
322 exceptions to this rule are largely the same compounds which deviated from the general trend for $323 \Delta_{f u s} H$.

Considering equation 1 , for a constant $T_{\text {fus }}$, a high value of $\Delta_{f u s} S$ should give a high $\Delta_{\text {fus }} H$, and so it is logical for $\Delta_{f u s} S$ and $\Delta_{f u s} H$ to correlate well. A plot of $\Delta_{f u s} S$ as a function of $\Delta_{f u s} H$ for the esters studied here, and the 53 esters from Acree and Chickos, ${ }^{21,22}$ results in a linear relationship with $\mathrm{R}^{2}=0.991$ (Figure 6). Long alkyl chains provide the opportunity for many chain-chain interactions, stabilizing the solid phase (high $\Delta_{f u s} H$ ), but also increase the entropy gain upon melting as they gain many degrees of freedom in the liquid phase.

\subsection{PCM Selection}

As described earlier, if $T_{f u s}$ is unchanged, high $\Delta_{f u s} S$ leads to high $\Delta_{f u s} H$. Therefore, when selecting a material for use as a PCM for thermal energy storage, increasing the linear alkyl chain length of the material can lead to higher energy storage density. (Although the theoretically more meaningful quantities are the molar enthalpy or entropy changes, in practical terms the gravimetric enthalpy change is more meaningful. This is why comparisons of isomers is particularly instructive.) While molar mass generally has a direct correlation with melting temperature, the distribution of the atoms is important. Within a series of materials with equal total linear alkyl chain length, materials with longer uninterrupted alkyl chains have higher $T_{f u s}$, which means that if $\Delta_{\text {fus }} S$ is unchanged, then $\Delta_{\text {fus }} H$ will be higher, and the material will have a higher thermal energy storage density. From Figure 3, the 26-carbon hexadecyl decanoate melts at a $\sim 20 \mathrm{~K}$ lower temperature, and has lower $\Delta_{f u s} H$, than the shorter and lighter 24-carbon ethyl docosanoate. The presence of the 22-carbon chain in the lighter compound results in the high melting point relative to the heavier compound containing only 10 and 16 carbon alkyl chains, despite having nearly equal $\Delta_{f u s} S\left(242\right.$ vs. $\left.244 \mathrm{~J} \mathrm{~mol}^{-1} \mathrm{~K}^{-1}\right){ }^{21,22}$ 


\section{Conclusions}

The interplay between molecular structure and thermodynamic properties is a valuable tool for PCM selection, and prediction of PCM properties. Increased molar mass leads to higher melting temperature, which in turn give rise to higher enthalpy change on fusion. Compact, symmetrical structures stabilize the solid phase and also give higher enthalpy changes. Materials with long alkyl chains have a high entropy change upon melting, and thereby also have high enthalpies of fusion.

In this study, the effect of carbon atom distribution in linear molecules of formula $\mathrm{C}_{12} \mathrm{H}_{24} \mathrm{O}_{2}$ on $T_{f u s}, \Delta_{f u s} H$, and $\Delta_{f u s} S$ was determined. Dodecanoic acid and three esters (hexyl

354 hexanoate, butyl octanoate, and ethyl decanoate) were analyzed by DSC. It was determined that 355 the highest melting temperatures and enthalpies of fusion could be achieved in the molecules 356 with the longest individual alkyl chains. As such, hexyl hexanoate had the lowest melting 357 temperature and enthalpy change, followed by butyl octanoate, ethyl decanoate, and finally 358 dodecanoic acid had the highest. Comparing with data compiled by Acree and Chickos ${ }^{21,22}$, this seems to be a general trend amongst the unbranched, saturated fatty esters. the ester data compiled from Acree and Chickos, ${ }^{21,22}$ with the highest values for $\Delta_{f u s} S$ typically

362 found for the isomers with the longest alkyl chains. The high entropy change gives rise to a high 363 enthalpy change on melting.

When selecting a PCM from long-chain organic molecules, longer chains, i.e., higher molar mass, will give higher melting points, and higher enthalpy changes and entropy changes on fusion. With regard to the main question asked here, namely "how does molecular structure

367 influence thermodynamic properties?", it was found that amongst isomeric ester PCMs, the 
368 highest $T_{f u s}, \Delta_{f u s} H$, and $\Delta_{f u s} S$ will be found for the ester with the longest individual alkyl chain.

369 Furthermore, fatty acids have higher melting temperatures and higher enthalpy changes than a

370 corresponding ester isomer. However, even the low-melting esters with evenly distributed carbon

371 atoms, such as hexyl hexanoate, have appreciable values of $\Delta_{f u s} H$ and could find use in

372 refrigeration and cooling applications.

373

374

375

376

377

378 Acknowledgements

379 M.A.W. is grateful to Professor R. J. C. Brown (Queen's University) and the late

380 Professor D. F. R. Gilson (McGill University) for useful discussions some decades ago

381 concerning thermodynamics of melting. The authors acknowledge the financial support of

382 NSERC (Discovery grant to M.A.W., NSERC CGS to J.A.N. and NSERC CREATE DREAMS

383 postdoctoral fellowship to S. K.) and assistance from Michel Johnson. This study also was

384 supported by the Canada Foundation for Innovation, the Atlantic Innovation Fund and other

385 partners that fund the Facilities for Materials Characterization at Dalhousie University. 


\section{References}

${ }^{1}$ U.S. Energy Information Administration. Residential Energy Consumption Survey. 2015, http://www.eia.gov/consumption/residential/

${ }^{2}$ Noël, J. A.; Kahwaji, S.; Desgrosseilliers, L.; Groulx, D.; White, M. A. Phase Change

Materials. In Storing Energy; Letcher, T., Ed.; Elsevier: Amsterdam, 2016; pp. 249-272.

${ }^{3}$ Zalba, B.; Marin, J. M.; Cabeza, L. F.; Mehling, H. Appl. Therm. Eng. 2003, 23, 251-283.

${ }^{4}$ Sharma, A.; Tyagi, V. V.; Chen, C. R.; Buddhi, D. Renew. Sust. Energy Rev. 2009, 13, 318345.

${ }^{5}$ Whitman, C. A.; M.B. Johnson, M B.; White, M. A. Thermochimica Acta 2012, 531, 54-59.

${ }^{6}$ A. Joseph, M. Kabbara, D. Groulx, P. Allred and M.A. White, Int. J. Energy Res. 40, 61-70 (2016).

${ }^{7}$ Arkar, C.; Medved, S. Sol. Energy. 2007, 81, 1078-1087. 
${ }^{8}$ https://www.phasechange.com/copy-of-enrg-blanket-tm

${ }^{9}$ Arkar, C.; Medved, S. Sol. Energy. 2007, 81, 1078-1087.

${ }^{10}$ Pielichkowska, K.; Pielichkowska, K. Prog. Mat. Sci. 2014, 65, 67-123.

${ }^{11}$ Desgrosseilliers, L.; Whitman, C. A.; Groulx, D.; White, M. A. Appl. Therm. Eng. 2013, 53, $37-41$.

${ }^{12}$ Kahwaji, S.; Johnson, M. B.; Kheirabadi, A. C.; Groulx, D.; White, M. A. Sol. Energy Mater. Sol. Cells. 2017, 167, 109-120.

${ }^{13}$ Brown, R. J. C.; Brown, R. F. C. J. Chem. Ed. 2000, 77, 724-731.

${ }^{14}$ Carnelley, T. Phil. Mag. 1882, 13, 112-130.

${ }^{15}$ Carnelley, T. Phil. Mag. 1882, 13, 180-193.

${ }^{16}$ Boese, R.; Kirchner, M. T.; Dunitz, J. D.; Filippini, G.; Gavezzotti, A. Helvetica Chimica Acta. 2001, 84, 1561-1577.

${ }^{17}$ Baeyer, A. Ber. Dtsch. Chem. Ges. 1877, 10, 1286-1288.

${ }^{18}$ Yang, K.; Cai, Z.; Jaiswal, A.; Tyagi, M.; Moore, J. S.; Zhang, Y. Angew. Chem. Int. Ed. 2016, $55,14090-14095$.

${ }^{19}$ Gilson, D. F. R. J. Chem. Ed. 1992, 69, 23-25.

${ }^{20}$ Handbook of Chemistry and Physics, $69^{\text {th }}$ ed.; CRC: 1988; pp. C666-C670.

${ }^{21}$ Acree, W.; Chickos, J. S. J. Phys. Chem. Ref. Data. 2016, 45, 03101.

${ }^{22}$ Acree, W.; Chickos, J. S. J. Phys. Chem. Ref. Data. 2017, 46, 013104.

${ }^{23}$ White, M. A.; Wasylishen, R. E.; Eaton, P.E.; Xiong, Y.; Pramod, K.; Nodari, N. J. Phys.

Chem. 1992, 96, 421-425.

${ }^{24}$ Walden, P. Z. Elektrochemie. 1908, 14, 713-724.

${ }^{25}$ White, M.A. Physical Properties of Materials, CRC Press (Boca Raton, FL) 2012. 
${ }^{26}$ Wei, L. L.; Ohsasa, K. ISIJ Int. 2010, 50, 1265-1269.

${ }^{27}$ Rozanna, D.; Chuah, T. G.; Salmiah, A.; Choong, T. S. Y.; Sa'ari, M. Int. J. Green Energy.

2005, 1, 495-513.

${ }^{28}$ Noël, J. A.; Allred, P. M.; White, M. A. Int. J. LCA. 2015, 20, 367-376.

${ }^{29}$ Kahwaji, S.; White, M.A. submitted.

${ }^{30}$ Gallart-Sirvent, P.; Martin, M.; Villorbina, G.; Balcells, M.; Sole, A.; Barrenche, C.; Cabeza,

L. F.; Canela-Garayoa, R. RSC Adv. 2017, 7, 24133-24139.

${ }^{31}$ Kahwaji, S.; Johnson, M. B.; Kheirabadi, A.; Groulx, D.; White, M. A. Appl. Energy. 2016, $168,457-464$.

${ }^{32}$ Rai, U. S.; Singh, O. P.; Singh, N. B. Can. J. Chem. 1987, 65, 2639-2642.

${ }^{33}$ Inagaki, T.; Ishida, T. J. Phys. Chem. C. 2016, 120, 7903-7915.

${ }^{34}$ Stamatiou, A.; Obermeyer, M.; Fischer, L. J.; Schuetz, P.; Worlitschek, J. Renew. Energy. 108, 401-409.

${ }^{35}$ Sabbah, R.; Xu-wu, A.; Chickos, J. S.; Planas Leitao, M. L.; Roux, M. V.; Torres, L. A. Thermochim. Acta. 1999, 331, 93-204.

${ }^{36}$ White, M. A.; Johnson, M. B. Thermal Methods. In Multi Length-Scale Characterization; Bruce, D. W.; O’Hare, D.; Walton, R. I., Eds.; John Wiley and Sons: West Sussex, UK, 2014; pp.63-120.

${ }^{37}$ Iwahashi, M.; Takebayashi, S.; Taguchi, M.; Kasahara, Y.; Minami, H.; Matsuzawa, H. Chem. Phys. Lipids. 2005, 133, 113-124. 
${ }^{38}$ Moreno, E.; Cordobilla, R.; Calvet, T.; Cuevas-Diarte, M. A.; Gbabode, G.; Negrier, P; Mondieig, D.; Oonk, H. A. J. New J. Chem. 2007, 31, 947-957.

Table 1. Thermodynamic properties of three esters and a fatty acid, each with chemical formula $\mathrm{C}_{12} \mathrm{H}_{24} \mathrm{O}_{2}$ * 


\begin{tabular}{|c|c|c|c|c|c|}
\hline Compound & $\boldsymbol{T}_{f u s} / \mathbf{K}$ & $\begin{array}{c}\Delta_{f u s} H / \\
\mathbf{k J ~ m o l}^{-1}\end{array}$ & $\begin{array}{c}\Delta_{f u s} \boldsymbol{H} / \\
\mathbf{J ~ g ~}^{-1}\end{array}$ & $\begin{array}{c}\Delta_{f u s} S / \\
\mathrm{J} \mathrm{mol}^{-1} \mathrm{~K}^{-1}\end{array}$ & $\begin{array}{c}\Delta_{f u s} S / \\
\mathbf{J ~ g ~}^{-1} \mathbf{K}^{-1}\end{array}$ \\
\hline Hexyl Hexanoate & $217 \pm 1$ & $22 \pm 1$ & $108 \pm 5$ & $100 \pm 5$ & $0.50 \pm 0.03$ \\
\hline Butyl Octanoate & $230 \pm 1$ & $28 \pm 1$ & $140 \pm 7$ & $122 \pm 6$ & $0.61 \pm 0.03$ \\
\hline Ethyl Decanoate & $252 \pm 1$ & $29 \pm 1$ & $143 \pm 7$ & $114 \pm 6$ & $0.57 \pm 0.03$ \\
\hline Dodecanoic Acid & $316 \pm 1$ & $36 \pm 2$ & $180 \pm 9$ & $114 \pm 6$ & $0.57 \pm 0.03$ \\
\hline
\end{tabular}

*Errors represent overall experimental uncertainties.

Figure 1. Entropies of fusion of 7038 organic compounds. Values from the data taken from Acree and Chickos. ${ }^{21,22}$ 
Figure 2. DSC traces showing melting for three 12-carbon linear esters and dodecanoic acid, taken at $2 \mathrm{~K} \mathrm{~min}^{-1}$. Endothermic peaks point down, and curves are displaced vertically for ease of viewing.

Figure 3. Melting temperatures for the linear, saturated esters as compiled by Acree and Chickos $^{21,22}$ and the present data. Among the esters for which data are available, at each carbon number, the ester with the longest individual alkyl chain has the highest $T_{f u s}$.

Figure 4. $\Delta_{\text {fus }} H$ for the linear, saturated esters as compiled by Acree and Chickos ${ }^{21,22}$ and present data.

Figure 5. $\Delta_{f u s} S$ for the linear, saturated esters as calculated from Acree and Chickos ${ }^{21,22}$ and present data.

Figure 6. Relationship between $\Delta_{f u s} S$ and $\Delta_{f u s} H$ for the linear, saturated esters as compiled from Acree and Chickos ${ }^{21,22}$ and present data. The line is a linear fit to the data. 


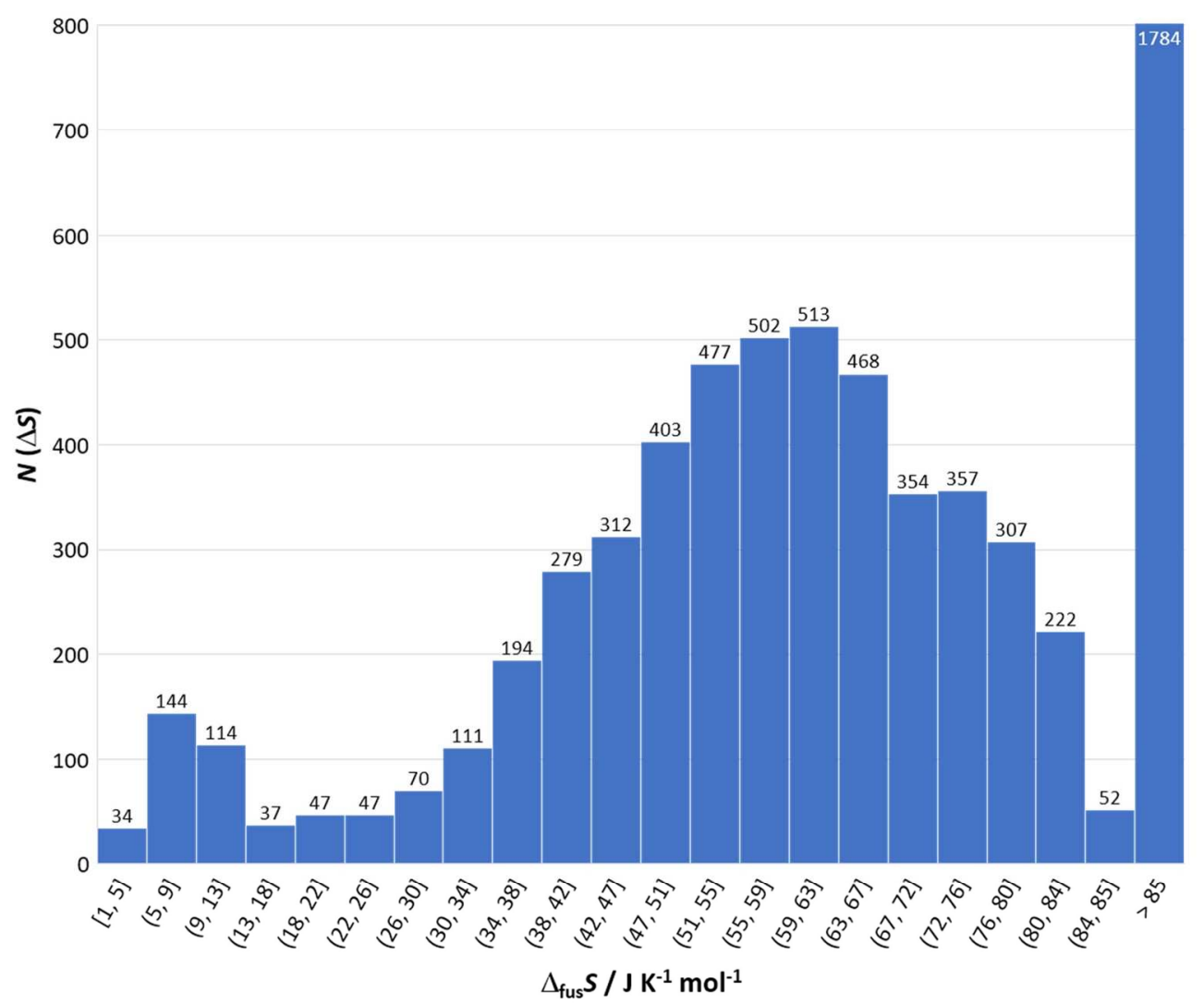

Figure 1. Entropies of fusion of 7038 organic compounds. Values from the data taken from Acree and Chickos.21,22

$712 \times 593 \mathrm{~mm}(96 \times 96 \mathrm{DPI})$ 


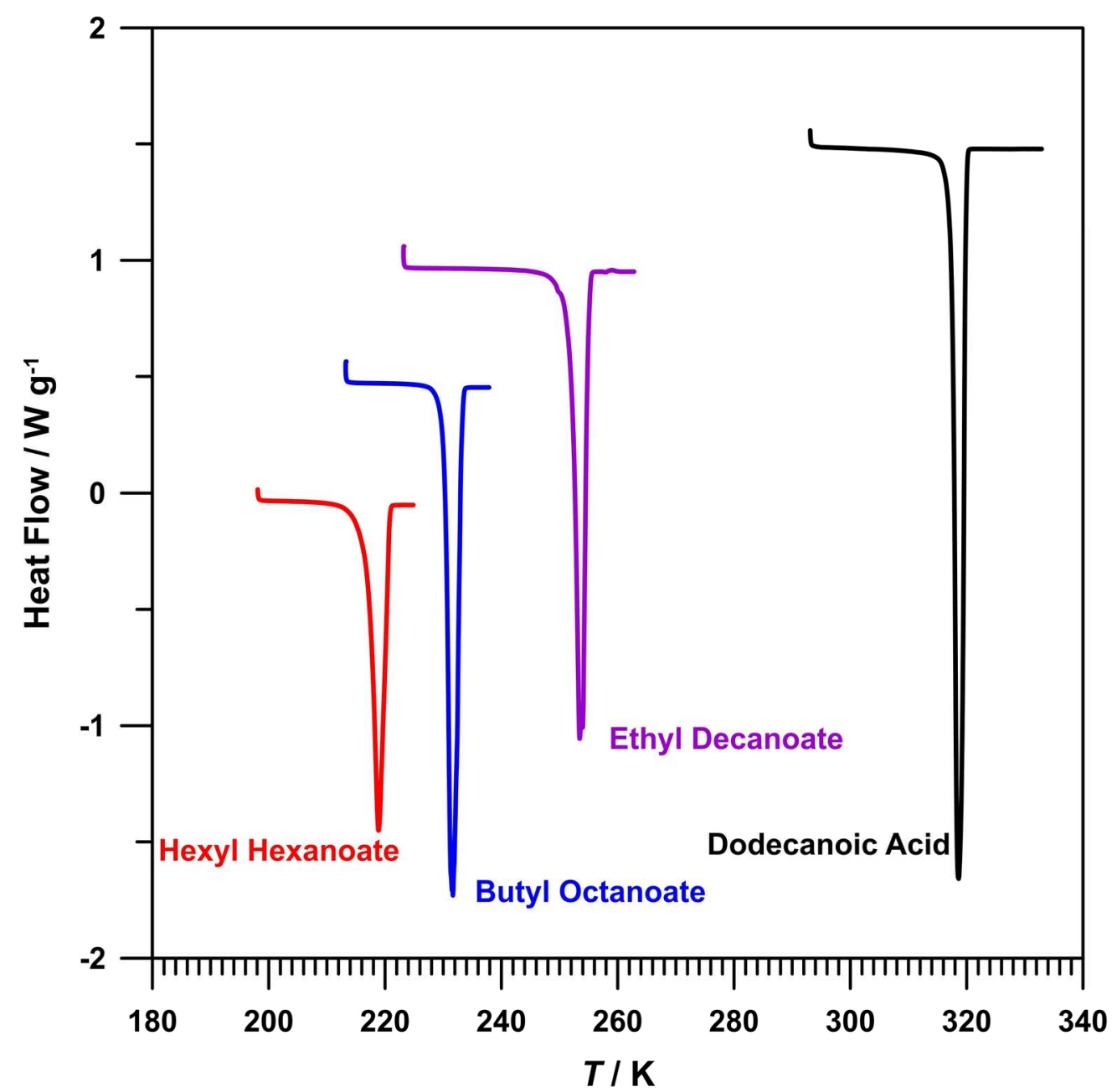

Figure 2. DSC traces showing melting for three 12-carbon linear esters and dodecanoic acid, taken at $2 \mathrm{~K}$ min-1. Endothermic peaks point down, and curves are displaced vertically for ease of viewing.

$177 \times 176 \mathrm{~mm}(300 \times 300 \mathrm{DPI})$ 


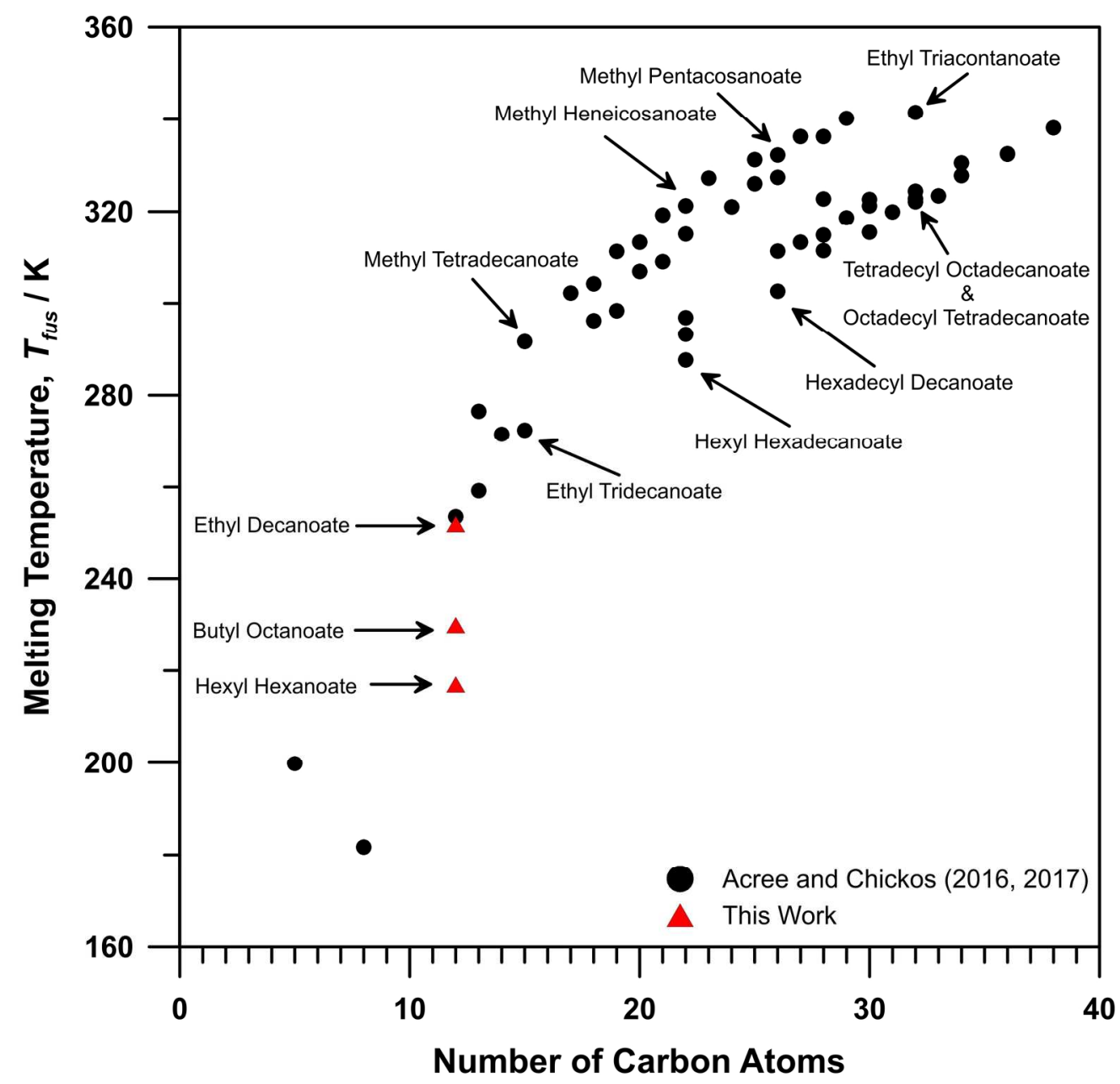

Figure 3. Melting temperatures for the linear, saturated esters as compiled by Acree and Chickos 21,22 and the present data. Among the esters for which data are available, at each carbon number, the ester with the longest individual alkyl chain has the highest Tfus.

$177 \times 172 \mathrm{~mm}(300 \times 300 \mathrm{DPI})$ 


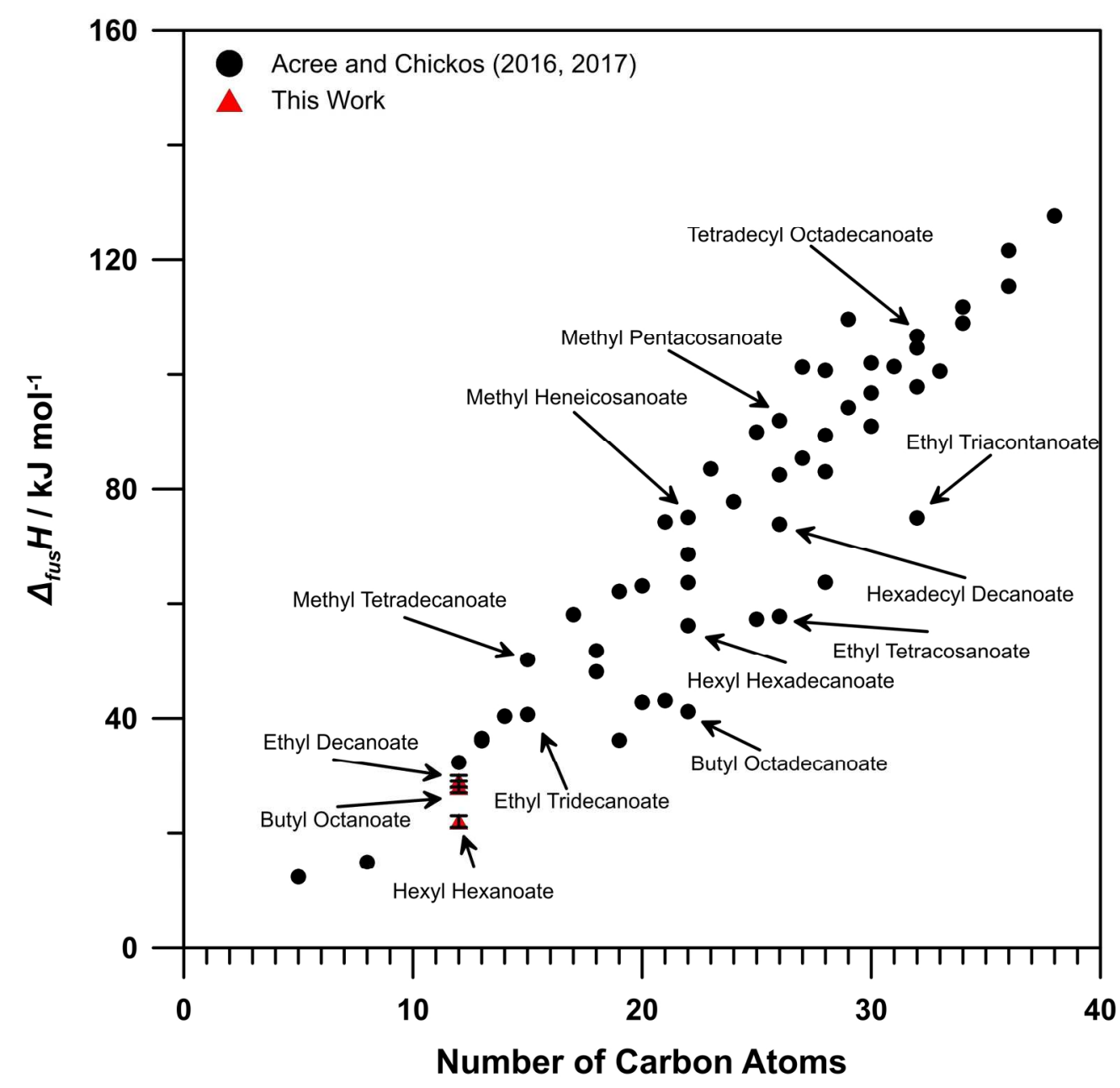

Figure 4. $\Delta$ fusH for the linear, saturated esters as compiled by Acree and Chickos21,22 and present data.

$177 \times 172 \mathrm{~mm}(300 \times 300 \mathrm{DPI})$ 


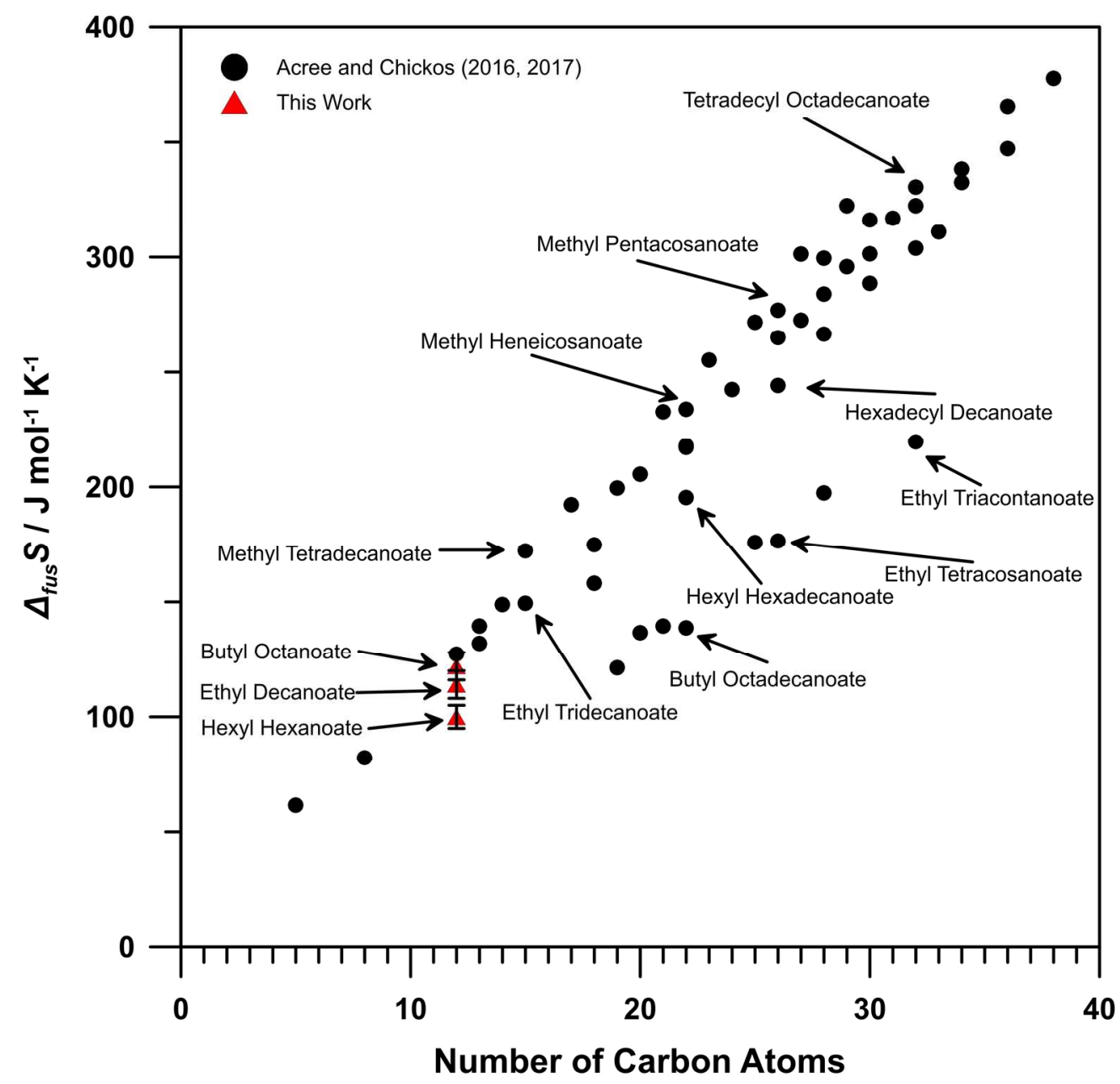

Figure 5. $\Delta$ fusS for the linear, saturated esters as calculated from Acree and Chickos21,22 and present data. $177 \times 172 \mathrm{~mm}(300 \times 300$ DPI $)$ 


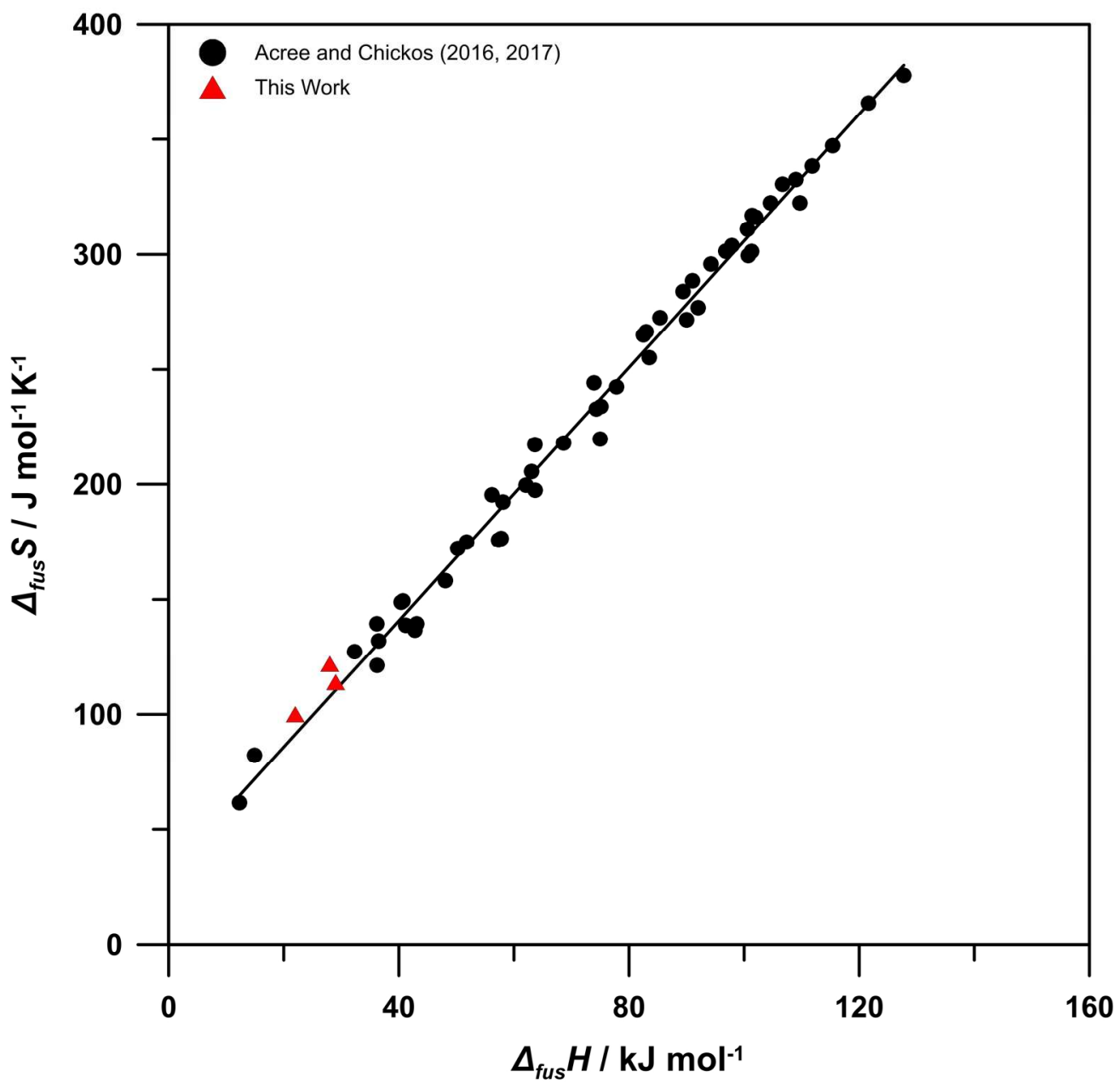

Figure 6. Relationship between $\Delta$ fusS and $\Delta$ fusH for the linear, saturated esters as compiled from Acree and Chickos21,22 and present data. The line is a linear fit to the data.

$178 \times 173 \mathrm{~mm}(300 \times 300 \mathrm{DPI})$ 